\title{
Swimming Performance: A Strategy to Evaluate Motor Dysfunction after Brain Ischemia in Mice
}

\author{
Claudia Gómez ${ }^{1}$, Martha Delgado-García ${ }^{1}$, Jacinto Santiago-Mejía ${ }^{1}$, Rosa Ventura-Martínez ${ }^{1}$, Leticia \\ Parra-Gámez ${ }^{2}$, Jacquelina González-Ríos ${ }^{2}$, Rodolfo Rodríguez ${ }^{1}$ \\ ${ }^{1}$ Department of Pharmacology, Faculty of Medicine, National University of Mexico, Mexico City, Mexico \\ ${ }^{2}$ Department of Anatomy, Faculty of Medicine, National University of Mexico, Mexico City, Mexico \\ Email: goac@servidor.unam.mx
}

Received October 15, 2013; revised November 19, 2013; accepted December 1, 2013

Copyright (c) 2013 Claudia Gómez et al. This is an open access article distributed under the Creative Commons Attribution License, which permits unrestricted use, distribution, and reproduction in any medium, provided the original work is properly cited.

\begin{abstract}
We have previously reported that sequential common artery sectioning (SCAS) in mice produces a reproducible pattern of mortality, extensive brain damage and a wide range of measurable neurobehavioral alterations that include motor incoordination and forelimb flexion. The present study describes a comprehensive method to assess motor functional outcome after brain ischemia produced by SCAS using swimming behavior. We found that after the second artery occlusion the time for completion of the swimming task significantly increased and the swimming pattern alterations observed in the ischemic mice showed no evidence of recovery (up to $96 \mathrm{~h}$ ). We view the swimming performance strategy described here as a sensitive, simple and economic procedure to assess motor performance after brain ischemia.
\end{abstract}

Keywords: Brain Ischemia; Motor Impairment; Mice; Motor Coordination; Swimming

\section{Introduction}

Experimental evaluation of motor performance in brain ischemic animals has become increasingly important in neuroprotective drug research, mainly because drugs that have proven effective in reducing infarct volume in animal stroke models have failed in human trails of acute ischemic stroke [1-4]. This lack of efficacy of putative neuroprotective drugs in clinical trails has highlighted the problem of translating experimental animal studies to the clinical setting, as well as the limitations of a purely histological approach in evaluating the effects of brain ischemia and neuroprotection. These shortcomings have questioned the validity of available animal models as preclinical tools in the study of neuroprotective agents [3-6]. In addition, the strategies employed in humans to identify the effects of brain ischemia and the degrees of neuroprotection have been rarely used in animal studies. While in most experimental studies the measurement of infarct size is considered essential for determining the effects of brain ischemia and recovery, clinical outcome and neuroprotection are almost invariably determined functionally and primarily by measures of motor performance. Thus, we consider that reliable measurements of motor function outcome in animals can provide meaningful data to determine the presence, severity and time course of impairments, as well as the consequences of neuroprotective drug administration. A major problem in functional output assessment is that traditional models of experimental stroke display a limited behavioral profile, and spontaneous recovery of motor deficits commonly occurs rapidly, especially after global cerebral ischemia [7,8]. In addition, in most cases it is necessary to use a variety of different tasks to examine the alterations induced by experimental stroke in rodents. Today, no single measurement technique has yet proven to be universally acceptable [6].

Previous studies from our laboratory have shown that sequential common carotid artery sectioning (SCAS) in mice produces a reproducible pattern of mortality, extensive brain damage and a wide range of measurable neurobehavioral alterations [9]. Of these, motor incoordination and forelimb flexion are the two most consistent neurological alterations after SCAS. The present study describes a comprehensive method to assess motor functional outcome after brain ischemia produced by SCAS using swimming behavior, a strategy that could be used to evaluate pharmacological neuroprotection. 


\section{Methods and Materials}

\subsection{Animals}

This study was performed in male CFW, middle-aged (37 - 40 weeks), mice weighing 40 - $50 \mathrm{~g}$ at the beginning of the experiments. Animals were obtained from our breeding facilities and housed 3 - 5 per cage (same litter) in a temperature controlled room $\left(22 \pm 2^{\circ} \mathrm{C}\right.$, relative humidity $55 \pm 3 \%$ ), with an automatically set time cycle of 12-h light/darkness (lights on 08:00 - 20:00). Food (Purina Chow, St. Louis, MO) and water were available ad libitum. Mice were allowed to acclimate to these environmental conditions for at least 1 week prior to experimentation. The experimental protocol was carried out under the provisions of the National Health Ministry guidelines for the use of laboratory animals (NOM 062ZOO-1999).

\subsection{Procedure}

Animals were randomly assigned to three different groups: experimental mice undergoing anesthesia and sectioning of both carotid arteries (SCAS), sham-operated mice undergoing anesthesia and full surgical procedures, except for artery ligation and sectioning, and mice not subjected to anesthesia or surgery (control group). Brain ischemia was produced as described previously [9]. Briefly, light anesthesia was induced with ether delivered through a facemask, and the left common artery was exposed through a midline neck incision and separated from the associated vagus and sympathetic nerves. The artery was sectioned between ligatures and the incision was closed with surgical thread. After surgery, animals were kept in a recovery room for about $1 \mathrm{~h}$ under heating lamps to maintain body temperature. Once animals regained complete consciousness, they were moved to the environmentally controlled room. Thirty-two days later, the right common carotid artery was sectioned using the procedure described above. Mice showing any degree of forelimb motor deficit were excluded from further experiments.

\subsection{Mortality}

Mortality was scored continuously over the first $6 \mathrm{~h}$ after the second surgery and then every $6 \mathrm{~h}$ during the first 24 h. Thereafter, mortality was scored every $12 \mathrm{~h}$ up to the end of the experiments (96 h).

\subsection{Neurobehavioral Testing}

We used the swimming performance test to quantify the neurobehavioral alterations produced by SCAS. We also identified a swimming pattern occurring in ischemic mice, which is consistent and potentially susceptible to manipulation by neuroprotective drugs. Neurobehavioral evaluations were performed immediately before and 24, 48, 72 and $96 \mathrm{~h}$ after both surgeries. All evaluations were performed at room temperature, between 10:00 and 14:00 h. Mice swimming performance was evaluated in dark conditions.

\subsection{Swimming Performance Test}

Swimming performance tests were conducted in a long narrow swimming pool made of clear acrylic (plexiglass). The pool was $80 \times 15 \times 25 \mathrm{~cm}$ and was filled with water $\left(18^{\circ} \mathrm{C}-20^{\circ} \mathrm{C}\right.$ ) to a depth of $10 \mathrm{~cm}$ (sufficient to prevent the mice form reaching the pool-floor). At one end of the pool was a platform (10 cm high, $9 \mathrm{~cm}$ diameter) onto which the animals could climb. Before the first surgery, mice were trained for five consecutive days, and the performance of each animal at day five was considered the baseline time. Regular food was withheld for 3 - 5 h before training and testing, but free access to water was maintained. A trial consisted of gently placing the animal at one end of the pool and allowing it to swim to the platform. Testing was made before each surgery (to have a baseline for each animal) and 24, 48, 72 and $96 \mathrm{~h}$ after the corresponding surgery. Each trial was recorded using a video recording system (OASPADVid OMNIALVA ${ }^{\mathrm{TM}}$ ) and the following measures of swimming performance were made: latency (time in seconds used to swim across the pool and climb with its four paws on the platform); distance (distance swum to reach the platform), velocity (swimming speed) and percentage of flotation (time spent without moving, still time).

\subsection{Swimming Pattern}

During each trial, a second camera was located to videotape the mice's swimming behavior from a lateral perspective. Each trial was recorded and played in slow motion for analysis. We identified the presence of 5 consistent behaviors: angle of swimming (horizontal or vertical with respect to the water line), straight forward swimming, pool-wall touching, touching the platform without climbing on it, and zigzag swimming.

\subsection{Data Analysis}

Survival curves were calculated with the Kaplan-Meier method and compared using the log-rank test. Statistical significance was assessed by analyses of variance (ANOVA) followed by Dunnett's test. For comparisons between SCAS and sham-operated animals Student's t-test for unpaired values was used. In all cases, a value of $\mathrm{p}<$ 0.05 was considered statistically significant.

\section{Results}

\subsection{Mortality}

No deaths were observed in control animals (no anesthe- 
sia, no surgery). Two deaths were observed in shamoperated mice (2/20). Therefore, sham-operated animals showed a $90 \%$ survival throughout the study period of 96 h. In the SCAS group, $23 \%$ of animals died within the first 15 min after the second surgery, and the number of deaths progressively increased to $37 \%$ at $24 \mathrm{~h}, 75 \%$ al 48 $\mathrm{h}$ and $80 \%$ at $96 \mathrm{~h}$. The results of the log-rank test indicated a highly statistically significant difference between these two curves ( $p<0.001$; Figure 1).

\subsection{Swimming Performance Test}

As reported by others, mice quickly learn to reach the platform and escape from the water [10-12]. By the end of the training period, it would take an animal an average of $6 \mathrm{~s}$ to reach the platform at the end of the swimming pool. After the first surgery, the time to reach the platform increased only at the $24 \mathrm{~h}$ observation in the SCAS group ( $\mathrm{p}<0.05$, when compared to the preoperative baseline). During the following observations (48, 72 and $96 \mathrm{~h}$ ), the time needed to reach the platform of all experimental groups was very similar to that of the preoperative baseline, except for the sham group, in which time reached statistical significance at 48 and $96 \mathrm{~h}$ when compared to the preoperative baseline ( $p<0.05$, Table 1). The swim distance increased in all experimental groups following the surgery but it only reached statisticcal significance in the SCAS group at $24 \mathrm{~h}$ observation ( $\mathrm{p}$ $<0.05$ compared to the baseline, Table 1). The swim speed of all three groups was very similar during the baseline observation, while after surgery it significantly decreased in the SCAS group at the 24 and $96 \mathrm{~h}$ when compared to the preoperative baseline $(p<0.05$, Table 1). During baseline observation all animals showed a similar still time, however animals of the control group reduced their flotation time at $48 \mathrm{~h}(\mathrm{p}<0.05$, Table 1$)$. Ischemic animals (SCAS group) showed a significant increase in their flotation time $96 \mathrm{~h}$ after the first surgery ( $\mathrm{p}$ $<0.05$, Table 1).

After the second surgery, the SCAS groups increased the time needed to reach the platform at all times but only significantly so at 24,48 and $72 \mathrm{~h}$ (p $<0.005$ when compared to the baseline and $\mathrm{p}<0.01$ when compared to the sham group, Figure 2(a)). The swim distance increased in the SCAS group at 24, 48 and $72 \mathrm{~h}$ after the second surgery ( $p<0.05$ when compared to the baseline, and $\mathrm{p}<0.01$ when compared to the sham group, Figure 2(b)). The sham group significantly increased the swim distance but only at $96 \mathrm{~h}$ after second surgery ( $\mathrm{p}<0.05$ when compared to baseline, Figure 2(b)). Animals from all three groups showed a constant and similar swim speed throughout the experiment; only animals from the sham group showed a significant increase at the $72 \mathrm{~h}$ observation ( $p<0.05$, Figure $2(\mathbf{c})$ ). The still time of SCAS animals decreased insignificantly after surgery.

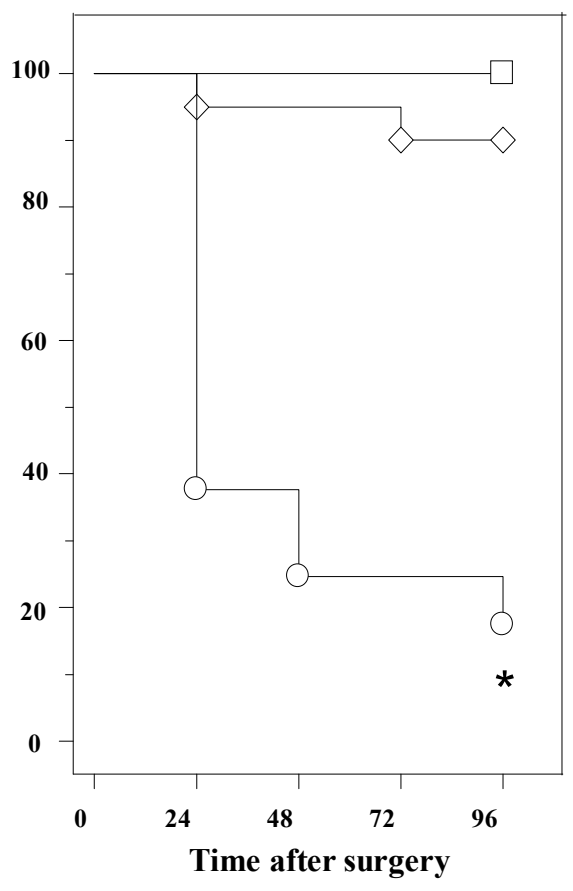

Figure 1. Survival curve in control $\square$, sham $\diamond$ and mice subjected to SCAS $\circ$. The survival curve was calculated by the Kaplan-Meier method and compared using log-rank test ( $<0.001$ ).

Table 1. Effect of first carotid artery sectioning (SCAS) on swimming performance. Data show mean results \pm SEM of the swimming performance scores obtained for each group (control, sham and SCAS) before (preoperative baseline) and $24,48,72$ and $96 \mathrm{~h}$ after the first surgery.

\begin{tabular}{ccccc}
\hline & & $\begin{array}{c}\text { Control } \\
(\mathrm{n}=11)\end{array}$ & $\begin{array}{c}\text { Sham } \\
(\mathrm{n}=22-27)\end{array}$ & $\begin{array}{c}\text { SCAS } \\
(\mathrm{n}=74-99)\end{array}$ \\
\hline Latency & Baseline & $6.80 \pm 1.10$ & $5.66 \pm 0.51$ & $5.21 \pm 0.35$ \\
(sec) & $24 \mathrm{~h}$ & $6.38 \pm 0.88$ & $5.50 \pm 0.40$ & $8.38 \pm 0.56^{*}$ \\
& $48 \mathrm{~h}$ & $6.28 \pm 1.79$ & $4.27 \pm 0.29^{*}$ & $5.91 \pm 0.50$ \\
& $72 \mathrm{~h}$ & $4.77 \pm 0.36$ & $4.81 \pm 0.22$ & $6.29 \pm 0.62$ \\
Distance & Baseline & $0.87 \pm 0.10$ & $0.80 \pm 0.05$ & $0.78 \pm 0.07$ \\
(m) & $24 \mathrm{~h}$ & $0.97 \pm 0.09^{*}$ & $0.87 \pm 0.04$ & $0.96 \pm 0.10$ \\
& $48 \mathrm{~h}$ & $1.13 \pm 0.33$ & $0.69 \pm 0.04$ & $0.83 \pm 0.09$ \\
Velocity & $72 \mathrm{~h}$ & $0.76 \pm 0.08$ & $0.72 \pm 0.04$ & $0.91 \pm 0.10$ \\
(m/s) & Baseline & $0.14 \pm 0.005$ & $0.15 \pm 0.005$ & $0.15 \pm 0.003$ \\
& $24 \mathrm{~h}$ & $0.14 \pm 0.01$ & $0.16 \pm 0.005$ & $0.13 \pm 0.006^{*}$ \\
& $48 \mathrm{~h}$ & $0.18 \pm 0.006$ & $0.16 \pm 0.007$ & $0.15 \pm 0.005$ \\
& $72 \mathrm{~h}$ & $0.15 \pm 0.012$ & $0.15 \pm 0.011$ & $0.16 \pm 0.005$ \\
& $96 \mathrm{~h}$ & $0.18 \pm 0.02$ & $0.17 \pm 0.011$ & $0.13 \pm 0.008^{*}$ \\
\% Flotation & Baseline & $24.97 \pm 3.18$ & $21.92 \pm 2.14$ & $22.33 \pm 1.09$ \\
& $24 \mathrm{~h}$ & $17.78 \pm 2.08$ & $16.17 \pm 1.25$ & $22.86 \pm 2.28$ \\
& $48 \mathrm{~h}$ & $11.31 \pm 1.91^{*}$ & $19.59 \pm 1.89$ & $21.89 \pm 1.84$ \\
& $72 \mathrm{~h}$ & $15.70 \pm 2.43$ & $22.25 \pm 3.51$ & $19.56 \pm 1.20$ \\
& $96 \mathrm{~h}$ & $22.06 \pm 5.44$ & $19.96 \pm 2.62$ & $32.76 \pm 2.75^{*}$ \\
\hline & & & &
\end{tabular}



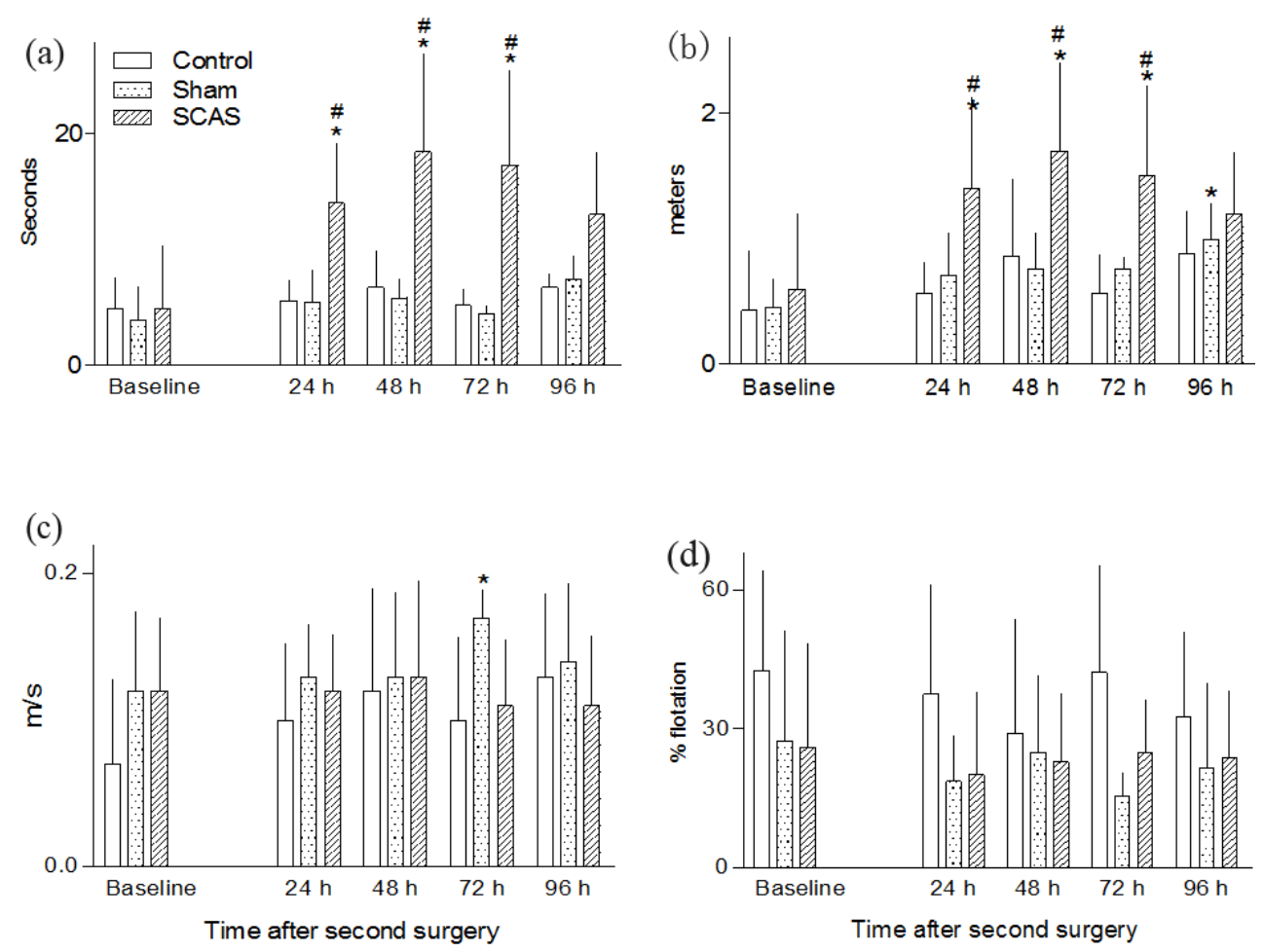

Figure 2. Effect of second artery sectioning in mice on swimming performance test. The top panel shows the effect of surgery in the latency to reach the platform (a) and the distance swam (b). The bottom panel shows the effect of the second surgery on swimming velocity (c) and on the percentage of flotation time (d). Each graph shows the mean values before (preoperative baseline) and 24, 48, 72 and $96 \mathrm{~h}$ after the second surgery. Data are derived from a variable number of surviving animals. White bars, non-operated animals (control $=11)$; dotted bars, sham operated mice $(n=27-20$, beginning and end of experiments) striped bars, animals subjected to common artery sectioning (SCAS, $n=99-20$, beginning and end of experiments). * $p<0.05$, compared with the corresponding preoperative value. ${ }^{\#} \mathbf{p}<0.05$, compared with the corresponding value of the control group.

Animals from the sham group showed a constant flotation time throughout the experiment (Figure 2(d)). Animals from the control group spent between 20 and $50 \%$ of the swimming time without moving (Figure 2(d)).

\subsection{Swimming Pattern}

As noted by others [13-15] during the training phase mice swim at an almost vertical angle to the water surface and use their forelimbs to stroke, but as they become familiar with the water environment and learn to reach the platform their swimming abilities improve and are able to reach the platform swimming at an horizontal angle in a completely forward direction with few forelimb strokes. After both surgeries, animals from the sham group showed a transient decrease in their swimming abilities: at $48 \mathrm{~h}$ post surgery they changed from a straight forward direction to a zig-zag line and increased the use of their forelimbs to stroke and touch the side walls of the pool, but by the end of the experiment (96 h) they showed a swimming pattern very similar to that of baseline (Figure 3(b)). At 72 h, some animals displayed a vertical angle with respect to the water surface, but at 96 $\mathrm{h}$ their swimming pattern was similar to that of control animals. After the first surgery, animals from the experimental group swam in a zig-zag line using their forelimbs for stroking and touching the walls of the pool.

Also, some animals lost their ability to swim horizontally but swan at a vertical angle during the four days of evaluation (data not shown). After the second surgery, animals from the experimental group showed an important modification of their swimming pattern: between 25 and $30 \%$ of them lost their ability to swim in a straight line and did so in a zig-zag line, with an important increase in the frequency (30\% - 40\%) of touching the side walls of the pool. At the $24 \mathrm{~h}$ post-surgery evaluation, $50 \%$ of the mice in this group used the forelimbs to stroke during swimming, and at the end of the experiment (72 and $96 \mathrm{~h}$ post-surgery evaluations) almost 100\% were using their forelimbs to stroke. At these intervals only a few animals ( $15 \%$ - 20\%) swam at a vertical angle to the water surface (Figure 3(c)).

\section{Discussion}

The procedure herein reported was developed to com- 


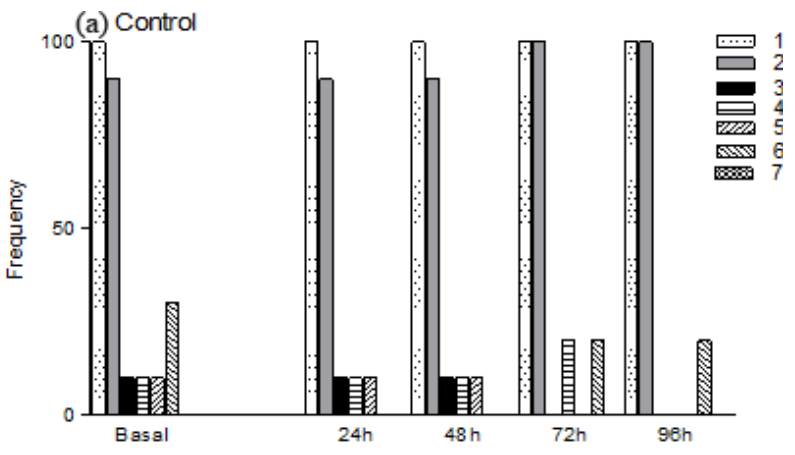

(b) Sham
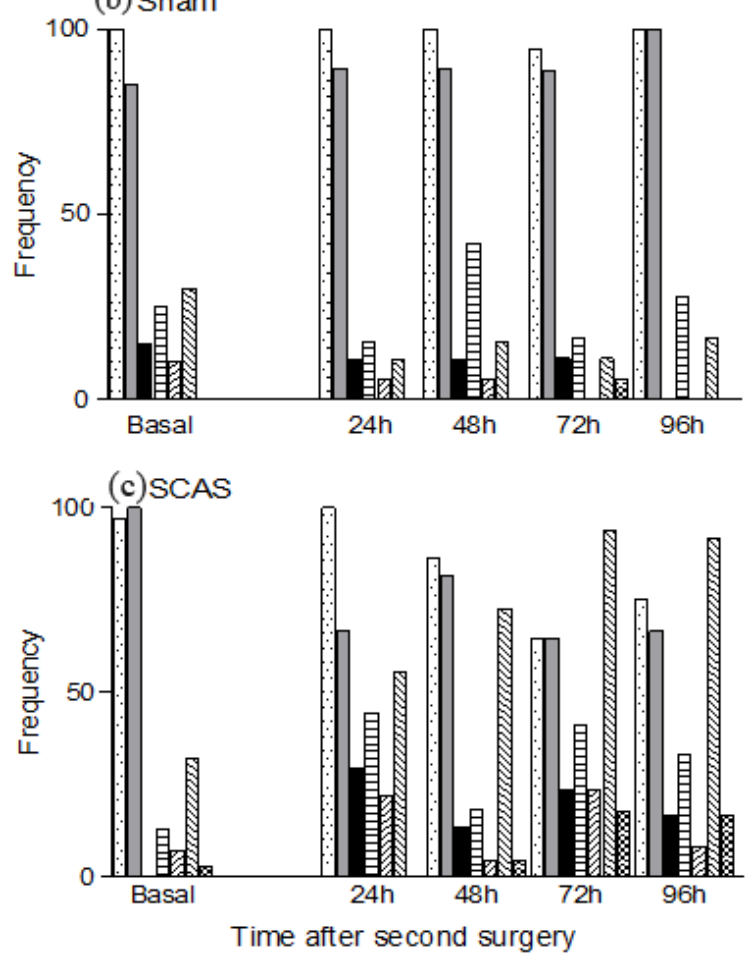

Figure 3. Frequency of the neurobehavioral alterations in swimming behavior detected throughout the $96 \mathrm{~h}$ period of observation in mice subjected to sequential carotid artery sectioning: 1) Swimming at a horizontal angle to the water surface; 2) Straight forward swimming, 3) Zig-zag swimming, 4) Pool-wall touching; 5) Touching the platform without climbing on it; 6) Hindlimb movement; and 7) Swimming at a vertical angle to the water surface. Animals were examined before (preoperative baseline) and 24, 48, 72 and $96 \mathrm{~h}$ after sthe econd surgery. Panel (a): Control animals $(n=11)$; panel $(b)$ : Sham operated mice $(n=27-20$, beginning and end of experiments); panel (c): animals subjected to common artery sectioning $(n=99-20$, beginning and end of experiments).

prehensively assess the functional state of each mouse before and after severe forebrain ischemia. For its development we considered: 1) That SCAS in mice typically results in severe tissue damage of a large portion of the brain, a consistent pattern of mortality and a wide variety of measurable neurobehavioral alterations [4]; 2)
The need for a full characterization of the motor functional deficits in ischemic mice; 3) The recognition that the effectiveness of pharmacological manipulations can be reliably determined only if severity of neurobehavioral deficits can be identified and quantified accurately at two or more points in time; and 4). The need for therapeutically relevant measures of neuroprotection

Our strategy to evaluate motor functional deficits after brain ischemia takes into account the following complementary features: A. Identification of the motor functional deficits in swimming performance induced by SCAS; B. Selection of the most frequent and relevant motor alterations produced by SCAS; and, C. The characterization of a swimming pattern induced by SCAS.

As reported by others, when mice are forced to swim for the first time, they display a wide variety of behaviors that reflect their anxiety and the need to escape from the water [16]. The swimming starts with uncoordinated movements of all four limbs and with their body in a vertical position with respect to the water line $[13,16]$. With successive trails mice modify their posture until they swim in a horizontal position with respect to the water line; they swim in a straight forward line and progressively stop using their forelimbs, regarding in their hindlimbs to propel their movement [12-14,16-18]. In our experiment, mice quickly learn to reach the platform and escape from the water, by the end of the training period, it would take an animal an average of $6 \mathrm{~s}$ to reach the platform at the end of the swimming pool (Table 1). After first surgery, ischemic mice showed a transient increase in latency to escape at the $24 \mathrm{~h}$ trail, but by the end of the test (96 h) they reached the platform as fast as they did during the baseline trail (Table 1). However, we found that the second artery occlusion significantly and consistently increased the time to reach the platform with no evidence of recovery (96 h, Figure 1). The inability to successfully reach the platform was apparently due to motor impairments in both their limbs: in their hindlimbs during swimming; and in their forelimbs during climbing of the platform. These observations, together with the results of previous studies showing that SCAS produces forelimb flexion with decreased resistance to lateral push and circling behavior, support the idea that in mice, SCAS produces a neurological deficit with hemiparesis or hemiplegia $[11,19]$. It is clear, however, that other factors, such as fatigue and/or decreased motivation to fulfill the task, that were not explored in this study, could also be involved in the increased time to reach the platform. In this study, with the use of a single procedure, we detected several neurobehavioral alterations in swimming behavior after SCAS. The swimming pattern consists of 6 well-defined and consistent behaviors: angle of swimming (horizontal or vertical to the water surface), straight forward swimming, forelimb flexion, pool-wall touching, 
touching the platform without climbing on it, and zigzag swimming. The total neurologic scores showed no evidence of recovery from these deficits but indicate some worsening over time. It is remarkable that except for a transient and slight increase in pool-wall touching observed in the sham animals, the surgical procedure did not induce significant neurobehavioral changes in this experimental group.

In most experimental studies, simple neurological scales modified from the original description of Bederson and colleagues [20] have been used to evaluate motor outcome after ischemic stroke. Other behavioral tests assessing motor deficits, including lack of coordination and partial paralysis after stoke do exist, but these have been primarily applied to rats [10,21]. In addition, one major problem of motor functional assessment is that traditional models of experimental stroke, such as middle cerebral artery occlusion (MCAO), display a somewhat limited behavioral profile, and spontaneous recovery of motor deficits commonly occur rapidly, so that it is generally necessary to employ a variety of different tasks to examine neurological alterations that occur in rodents after experimental stroke.

The swimming pattern described in this article delineates six major deficits that can be attributed to brain ischemia. Our proposal provides a quantitative measurement of the degree of neurological deficit after brain ischemia, and at the same time, allows for periodic comparisons within or among groups of animals. The relatively high level of neurobehavioral dysfunction and the remarkable difference between sham-operated and SCAS animals offer the possibility to test treatment effects and to monitor progress of deficits over time in groups of animals.

\section{Conclusion}

In conclusion, this study describes a simple, consistent and efficient strategy to evaluate motor deficits induced in mice by acute ischemia using swimming behavior as an indicator. We believe that this procedure can be useful for neuroprotection studies and allows monitoring the progress of deficits over time in groups of animals.

\section{Acknowledgements}

This study was supported by CONACyT and DGAPA.

We thank Mr. Constantino Ramirez Miranda for his excellent technical assistance.

The authors declare that they have no conflict of interest.

\section{REFERENCES}

[1] M. Ginsberg, "Neuroprotection for Ischemic Stroke: Past,
Present and Future,” Neuropharmacology, 2008.

[2] A. R. Green and A. Shuaib, "Therapeutic Strategies for the Treatment of Stroke,” Drug Discovery Today, Vol. 11, No. 15/16, 2006, pp. 681-693. http://dx.doi.org/10.1016/j.drudis.2006.06.001

[3] J. Minnerup, B. A. Sutherland, A. M. Buchan and C. Kleinscmitz, "Neuroprotection for Stroke: Current Status and Future Perspectives," International Journal of Molecular Science, Vol. 13, 2012, pp. 11753-11772. http://dx.doi.org/10.3390/ijms130911753

[4] V. E. O’Collins, M. R. Macleod, G. A. Donnan, L. L. Horky, B. H. van der Worp and D. W. Howells, "1026 Experimental Treatments in Acute Stroke,” Annals of Neurology, Vol. 59, No. 3, 2006, pp. 467-477. http://dx.doi.org/10.1002/ana.20741

[5] G. A. Donnan, "Acute Stroke Therapies: Some Unexpected Challenges,” International Journal of Stroke, Vol. 2, No. 4, 2007, pp. 235-239. http://dx.doi.org/10.1111/j.1747-4949.2007.00156.x

[6] I. M. Macrae, "Preclinical Stroke Research-Advantages and Disadvantages of the Most Common Rodent Models of Focal Ischaemia,” British Journal of Pharmacology, Vol. 164, 2011, pp. 1062-1078.

[7] F. Block, "Global Ischemia and Behavioral Deficits," Progress in Neurobiology, Vol. 58, 1999, pp. 279-285.

[8] J. Lubjuhn, A. Gastens, G. von Wilpert, P. Bargiotas, O. Hermann, S. Murikinati, T. Rabie, H. Marti, I. Amend and T. G. Hampton, "Functional Testing in a Mouse Stroke Induced by Occlusion of the Distal Middle Cerebral Artery,” Journal of Neuroscience Methods, Vol. 184, No. 1, 2009, pp. 95-103. http://dx.doi.org/10.1016/j.jneumeth.2009.07.029

[9] R. Rodríguez, I. Rodríguez-Boscán, D. Lugo-Sira, H. Vidrio, E. Hong, M. Medina and I. Herrera, "Bilateral Sequential Common Carotid Artery Sectioning in Mice as a New Model for Testing Neuroprotective Drugs,” Journal of Stroke and Cerebrovascular Diseases, Vol. 9, No. 1, 2000, pp. 45-53. http://dx.doi.org/10.1016/S1052-3057(00)33693-X

[10] R. Haobam, K. M. Sindhu, G. Chandra and K. P. Mohanakumar, "Swim-Test as a Function of Motor Impairment in MPTP Model of Parkinson's Disease: A Comparative Study in Two Mouse Strains,” Behavioral Brain Research, Vol. 163, No. 2, 2005, pp. 159-167. http://dx.doi.org/10.1016/j.bbr.2005.04.011

[11] R. Rodríguez, J. Santiago-Mejía, C. Gomez, E. RamírezSanJuan, "A Simplified Procedure for the Quantitative Measurement of Neurological Deficits after Forebrain Ischemia in Mice,” Journal of Neuroscience Methods, Vol. 147, No. 3, 2005, pp. 22-28. http://dx.doi.org/10.1016/j.jneumeth.2005.02.013

[12] I. Q. Whishaw, J. C. Cassel and L. E. Jarrard, "Rats with Fimbria-Fornix Lesions Display a Place Response in a Swimming Pool: A Dissociation between Getting There and Knowing Where,” Journal of Neuroscience, Vol. 15, No. 8, 1995, pp. 5779-5788.

[13] A. Bekoff and W. Trainer, "The Development of Interlimb Coordination during Swimming in Postnatal Rats," Journal of Experimental Biology, Vol. 83, 1979, pp. 1-11. 
[14] L. Enthoven, S. Dalm, E. R. de Kloet and M. S. Oitzl, "Swim Posture of Mice Does Not Affect Performance in the Water Maze,” Brain Research, Vol. 1003, No. 1-2, 2004, pp. 36-41.

http://dx.doi.org/10.1016/j.brainres.2003.10.074

[15] E. Gisel, M. Gruenthal and S. Finger "Swimming as a Method for Assessing Motor Cortex Integrity in the Rat," Brain Research, Vol. 239, 1982, pp. 649-654.

[16] T. H. J. Burne, A. N. B. Johnston, J. J. McGrath and A. Mackay-Sim, "Swimming Behavior and Post-Swimming Activity in Vitamin D Receptor Knockout Mice," Brain Research Bulletin, Vol. 69, No. 1, 2006, pp. 74-78. http://dx.doi.org/10.1016/j.brainresbull.2005.10.014

[17] S. Stoltz, J. L. Humm and T Schallert, "Cortical Injury Impairs Contralateral Forelimb Immobility during Swimming: A Simple Test for Loss of Inhibitory Motor Control," Behavioural Brain Research, Vol. 106, No. 1-2, 1999, pp. 127-132.

http://dx.doi.org/10.1016/S0166-4328(99)00100-X
[18] I. Q. Whishaw, A. J. Nonneman and B. Kolb, "Environmental Constraints on Motor Abilities Used in Grooming, Swimming and Eating by Decorticate Rats," Journal of Comparative Physiology Psychology, Vol. 95, No. 5, 1981, pp. 792-804. http://dx.doi.org/10.1037/h0077835

[19] C. Gomez, J. Santiago-Mejía, R. Ventura-Martínez and R. Rodríguez, "The Sunflower Seed Test: A Simple Procedure to Evaluate Forelimb Motor Dysfunction after Brain Ischemia,” Drug Development Research, Vol. 67, No. 9, 2006, pp. 752-756. http://dx.doi.org/10.1002/ddr.20117

[20] J. B. Bederson, L. H. Pitts, M. Tsuji, M. C. Nishimura and H. Bartkowski, "Rat Middle Cerebral Artery Occlusion: Evaluation of the Model and Development of a Neurologic Examination,” Stroke, Vol. 17, 1986, pp. 472476. http://dx.doi.org/10.1161/01.STR.17.3.472

[21] A. C. DeVries, R. J. Nelson, R. J. Traystman and P. D. Hurn, "Cognitive and Behavioral Assessment in Experimental Stroke Research: Will It Prove Useful?” Neuroscience Biobehavior Reviews, Vol. 25, 2001, pp. 325-342. 